\title{
Distributed Multiple Target Tracking Protocol in Two- Dimensional Binary Sensor Networks
}

\author{
Kuei-Chieh Lin and Chun-Shien Lu \\ Institute of Information Science, Academia Sinica, Taipei, Taiwan
}

\begin{abstract}
In this paper, a distributed multiple target tracking protocol (DMTTP) is proposed for target counting and trajectory estimation in two-dimensional binary sensor networks under the ideal sensing model. We first propose to formulate the minimum target number problem as a minimum clique partition problem and then investigate the performance limit of binary sensor networks in counting multiple targets in a two-dimensional sensing field. In DMTTP, a positive clique must be maintained in time for target counting. Consequently, we present two strategies to solve the two puzzles raised in the use of spatiotemporal information. For the target trajectory estimation task, an algorithm, operating under the ideal sensing range, is proposed. Our algorithm is designed to be light to decrease the load of each sensor so as to increase the efficiency of the sensor network.
\end{abstract}

Keywords: Binary Sensing, Clique Partition, Distributed Algorithms, Sensor Networks, Target Counting, Target Tracking

\section{Introduction}

The applications of target tracking include many kinds of location-based services, such as pursuit evasion games, indoor or outdoor surveillance, search and rescue, and even disaster response system, along with being able to help many kinds of spatial-temporal data collection. Nowadays, using sensor networks to track targets is an important issue that has been extensively studied. To keep the efficiency and reliability of a sensor network system, there are a lot of sensor nodes with limited resources required to be deployed in a monitoring field.

In this paper, we adopt the binary sensor, a kind of sensor that produces only one bit information to represent the presence or absence of a target in the sensing range. Since it only provides very restricted monitoring information, the communication overhead can be reduced, and we can deal easily with the sensor fault and communication noise. The limited information available, however, poses challenges on utility of such kind of sensor.

In this paper, we begin by studying the performance limit of target counting in a two-dimensional binary sensor network by modeling the minimum target number problem as a minimum clique partition problem. We then find that spatial information is too rough to be suitable for target counting. To address this problem, we adopt spatiotemporal information and propose an idea of maintaining each positive clique in time. This can work well except in the situation where targets move closely, which will raise two puzzles.

Consequently, we develop a Distributed Multiple Target Tracking Protocol (denoted by DMTTP) using spatiotemporal information, which is feasible for target counting and trajectory estimation. For the target counting task, DMTTP, which contains two strategies used to solve the aforementioned two puzzles, is designed to be executed by only communicating with neighboring sensors whose sensing ranges overlap. For target trajectory estimation, we use the average distance between any two triggers ${ }^{1}$ or in any sampling time interval as the result of distance estimation of the target trajectory. In particular, DMTTP is designed to be adaptive in a sensor network with ideal sensing range (commonly-used deterministic disk sensing model). Moreover, DMTTP is designed to

\footnotetext{
${ }^{1}$ Trigger of a sensor is defined to respond as either positive-edge or negative-edge trigger.
} 
be light to decrease the load of each sensor so as to increase the efficiency of the sensor network.

For evaluation of the proposed protocol, DMTTP, we show through extensive simulations that our pithy algorithms can yield more accurate estimations than two competing methods [11], [13], which are based on particle filter.

\section{Related Work}

Generally, targets can be classified in two categories, according to their shapes. Different types of targets need specific methods for efficient tracking. The first category is the continuous object, such as diffused poison gas or biochemical and chemical liquids, which can spread through a very wide area. For this kind of object, with the characteristic of diffusion, increasing size, changing shape and so on, some tracking approaches [1] [2] have been presented. The second category is the individual object, such as intruders and vehicles, which is usually very small in size, compared to the area where the sensor network is deployed. This kind of target can be further divided into two types: single target and multiple targets.

In the literature, several prior works [3] [5] [6] extensively studied the problem of tracking an individual target in the environment where multiple targets exist. Many kinds of sensing modules, such as acoustic and seismic sensors [7], have been utilized for the tracking task. Some kinds of sensors can provide richer information and wider sensing ranges. If sensor networks, however, are built based on dense deployment of disposable and lowcost sensor nodes [8], the aforementioned sensors may not be practical due to their high price. Therefore, target tracking using binary sensors is considered a feasible direction.

As binary sensor networks are somewhat restricted in finding either the accurate number of targets or the exact location of each target due to the very limited monitoring information provided, many researchers have devoted time to solving these problems. Toward the target counting problem, Singh et al. [13] proposed determining the lower bound on the target number in a one-dimensional field, which is known as the maximum independent set problem, and provided a greedy algorithm to solve it. Moreover, Gandhi et al. [4] studied this problem under a wide variety of binary sensing models. Toward the problem of target location, earlier works have dealt with single target tracking [9][10]. In particular, the fundamental limit of spatial resolution in target tracking was proven in [11]. Recently, the methods based on Kalman filter [12] and particle filter [13] have been popular for multiple target tracking. These methods can effectively solve the nonlinear estimation problem but also incur considerable computation overhead.

\section{Our Findings in Target Counting}

In this section, we first study the performance limit of target counting in a two-dimensional binary sensor network using spatial information only. To make the discussions in this section more concise, we assume a kind of ideal sensor is used. Each sensor's sensing range is a circular disk. An ideal sensor will output 1 whenever a target is within its sensing range; otherwise 0 is output. It should be noted that we currently focus on the ideal sensing range (commonly-used deterministic disk sensing model), as described in Sec. 4.

Then, we propose to transfer the target tracking problem from the space domain to the spatiotemporal domain in order to earn more information and make the problem more tractable. We investigate the conditions where binary sensor networks can or cannot count the number of targets precisely. We address two puzzles in counting targets when using spatiotemporal data. To resolve these puzzles, we develop a distributed multiple targets tracking protocol (DMTTP), described in Section 4.

\subsection{Performance Limit of Target Counting Using Spatial Information}

Since the nature of a binary sensor only reveals the existence of nearby targets, but not the number of targets, we assume at most one target appears in the sensing range of a binary sensor in our theoretic analyses. It is not difficult to count targets when targets are distant enough from each other, e.g., 4 times of the radius of sensing 
range [13]. Nevertheless, once several targets move close together so that the sensing ranges of sensors, which output 1 , comprise a large and continuous region, counting targets accurately becomes a complex problem.

We describe our method starting from a basic idea on a binary sensor network with only spatial information available.

Theorem 1. Consider a set of binary sensor nodes $s_{1}, s_{2}, \ldots, s_{n}$ with sensing range $S_{1}, S_{2}, \ldots, S_{n}$, respectively. When $S_{1} \cap S_{2} \cap \ldots \cap S_{n} \neq \phi$ and all $s_{i}$ 's $(i=1,2, \ldots, n)$ output 1 , the number of targets in the region $S_{1} \cup S_{2} \cup \ldots \cup S_{n}$ is uncertain and at least 1 .

We now understand from Theorem 1 that it is impossible to count targets exactly in binary sensor networks, and it seems that this number can be extremely large. Hence, we turn to seek a lower bound on the number of targets. We define a set of sensor nodes, which output 1 and the sensing ranges of any two of them overlap, as a positive clique. The lower bound on the number of targets can be found according to Theorem 2 .

Theorem 2. Given a binary sensor network deployed in a two-dimensional field, where a group of sensors output 1 at a certain time and uncertain numbers of targets are inside their sensing ranges, a lower bound on the number of targets is the minimum number of positive clique partitions.

In [13], Singh et al. presented that the minimum number of targets can be bounded by the maximum set of positively independent sensors, which are defined for any two sensors satisfying: (i) their outputs are 1, and (ii) either their sensing ranges are disjointed or overlap but the overlap is covered by other sensors, which output 0 . We find that this estimation is not very accurate in some cases. One example is when more than three nodes which output 1 are arranged as a cycle of odd order. In such a situation, the minimum number of positive clique partitions is larger than the maximum number of positively independent sensors, which yields more accurate results in target counting.

Based on the above observations, we seek a solution extended from Theorem 2 to explore the capability of a binary sensor network in target counting in Theorem 3, without resorting to solving minimum clique partitions that is known to be NP-hard.

Theorem 3. Consider a set of binary sensor nodes $s_{1}, s_{2}, \ldots, s_{n}$, with sensing range $S_{1}, S_{2}, \ldots, S_{n}$, respectively, and each sensing radius is $r$. If the set of sensor nodes fully covers an area A, the maximum number of targets that can be identified is $A / \pi r^{2}$.

Now, we understand that the capability of target counting in a binary sensor network is affected by the sensing range (i.e., $r$ in Theorem 3) of each sensor, and the increase of density of sensor nodes cannot help in releasing this limit. Moreover, the methods for counting targets based on spatial information alone, like minimum clique partitions or maximum independent sets [13] [16][17], can only provide a very rough estimation. Such kind of results is useless for estimating the target trajectory since we still cannot get the true number of targets. Even if the correct number of targets can be obtained, it is also very difficult to obtain the actual location of each target. Consequently, we propose a new scheme based on the use of spatiotemporal information.

\subsection{Target Counting Using Spatiotemporal Information}

To achieve actual target counting, rather than just estimating a range of number of targets, we propose to monitor and modify all positive cliques on the go from analyzing the spatiotemporal information. Note that the assumption that a target can trigger only one sensor at a time and a sensor cannot be triggered by more than one target simultaneously, to our knowledge, is common for all the existing methods and is also made in this paper. When a sensor gets a positive-edge trigger, it will be added into a certain positive clique. Any sensor getting a negativeedge trigger will be deleted from the positive clique that contains it. This idea tackles the cases with an arbitrary number of targets moving randomly in a sensing field except when targets move closely (but not in the same positive clique), which may cause two problems. They are described but somewhat simplified below. They can, however, be extended to more complex problems involving arbitrary number of targets and sensors.

Problem 1. Consider two targets moving in a binary sensor network, as illustrated in Figure 1. At to (Figure1(a)), 
Sensors " $a$ " and " $c$ " detect Targets " 1 " and "2," respectively, so that we have two positive cliques, $\{a\}$ and $\{c\}$. If Sensor " $b$ " is triggered (Figure 1(b)), how do we ascertain which target is detected by " $b$ "?

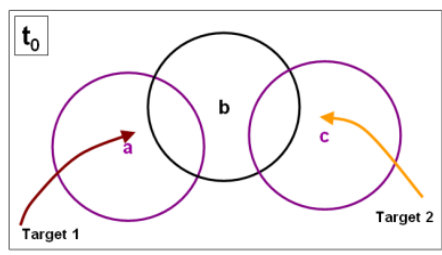

(a)

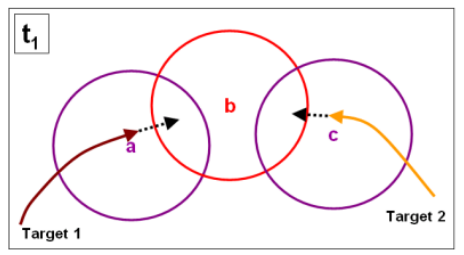

(b)

Fig. 1. Illustration of Problem 1.

Problem 2. Consider another scenario illustrated in Figure 2. Figure 2(a) shows that Sensor " a" detects Target " 1 ," and both Sensors " $b$ " and "c" detect Target "2" at $t_{0}$. We now have $\{a\}$ and $\{b, c\}$ as positive cliques. Then, Target " 1 " enters the overlap of sensing ranges of " $a$ " and " $b$ " at $t_{1}$, as shown in Figure 2(b). At this time, positive cliques are still maintained to be $\{a\}$ and $\{b, c\}$ because Sensor " $b$," currently being triggered by Target "2," cannot be triggered again. While at $t_{2}$ Target "2" has left the sensing range of " $b$ ", as shown in Figure 2(c), but Sensor " $b$ " cannot know which target ("1" or "2") triggers it. Therefore, the positive cliques are still preserved to be $\{a\}$ and $\{b, c\}$ although they should be $\{a, b\}$ and $\{c\}$ at $t_{2}$, instead.

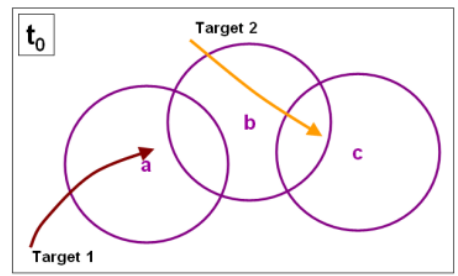

(a)

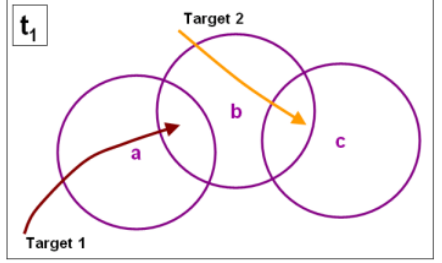

(b)

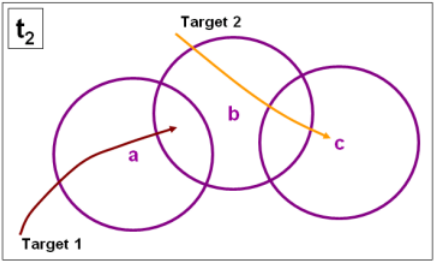

(c)

Fig. 2. Illustration of Problem 2.

Problem 1 occurs when targets are moving toward each other. In a general case, we need to find a positive clique and assign the newly triggered sensor to update it. In Figure 1(b), however, we can find two possible positive cliques, which leads to ambiguity. On the other hand, Problem 2 occurs when one target tends to follow another one. Since a sensor cannot be triggered while one target is within its sensing range, no new triggers occur and cliques are kept unchanged, as shown in Figures 2(b), (c). We can see that the positive cliques will be false (either the number of cliques or the range of a clique is erroneous), leading to erroneous target trajectory estimation, when the two aforementioned problems occur. In addition, erroneous positive cliques will also cause inaccurate estimation of positive cliques and, thus, lead to inaccurate estimation of targets.

\section{Distributed Multiple Target Tracking Protocol}

To realize target tracking in a binary sensor network, we design a distributed multiple target tracking protocol (denoted by DMTTP). The following subsections describe DMTTP in depth. We first introduce the basic ideas behind DMTTP. Then, we give an explanation of its operation when different events occur.

\subsection{Basic Ideas for DMTTP}

As we have mentioned in Section III.A, the number of positive cliques can be seen as the number of targets in a binary sensor network. In order to count the number of targets as well as trace the targets, DMTTP needs to maintain each positive clique in time. Consequently, we must solve the two problems described in Section III.B. Note that the two problems and their corresponding strategies share the same setting.

To tackle Problem 1, we present a strategy which we call "detain suspect." The initial condition at $\mathrm{t}_{0}$ is the same as in Problem 1. At $t_{1}$, Target " 1 " enters the sensing range of Sensor "b" (Figure 3(a)). Nevertheless, at this time instant, which of the targets (Target 1 or Target 2) has actually entered the sensing range of " $b$ " is unknown. Therefore, " $b$ " is temporarily detained instead of adding in either $\{a\}$ or $\{c\}$ for update at $t_{1}$. At $t_{2}$, Target " 2 " 
enters the sensing range of Sensor "d" and "d" is triggered (Figure 3(b)). Since Sensor "d" can only be assigned to the Positive clique $\{c\}$ to become $\{c, d\}$, Sensor " $b$ " can now be released and assigned to the Positive clique $\{a\}$ to become $\{a, b\}$.

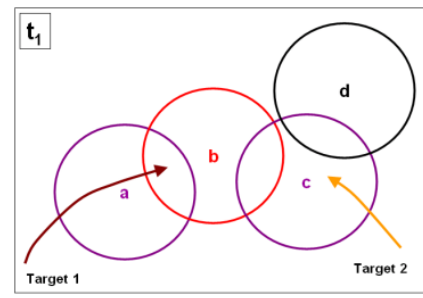

(a)

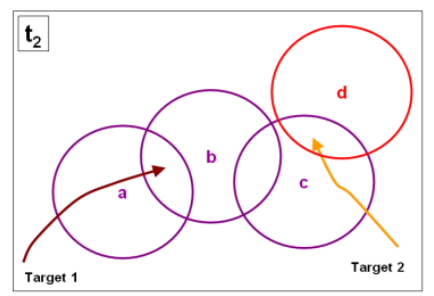

(b)

Fig. 3. Strategy 1: Detain suspect.

To tackle Problem 2, we present a strategy called "illegal address and reapplying." The initial condition at $t_{0}$ is the same as Problem 2. Then, Target " 1 " enters the sensing range of Sensor "b" at $t_{1}$ (Figure4(a)) and Target " 2 " leaves Sensor " $b$ " at $t_{2}$ (Figure 4(b)). At this moment in time, the positive cliques, $\{4 a\}$ and $\{b, c\}$, are, respectively, reported just as what they were at $\mathrm{t}_{0}$. This fault can be fixed when Target "2" enters the sensing range of Sensor "d" (Figure 4(c)). Since it is impossible for a positive clique to be $\{b, c, d\}$ in Figure 4(c), we check every sensor in $\{b, c, d\}$ for a sensor that can be added in $\{a\}$ for updating cliques. Finally, Sensor " $b$ " is found and new positive cliques of $\{a, b\}$ and $\{c, d\}$ are reapplied.

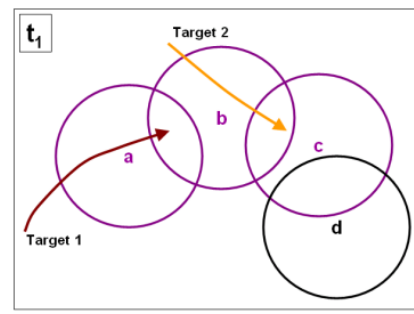

(a)

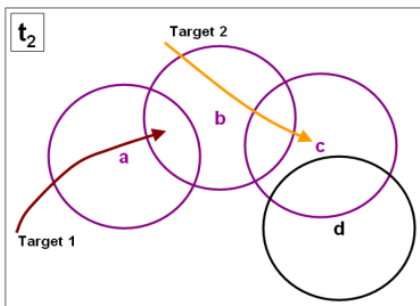

(b)

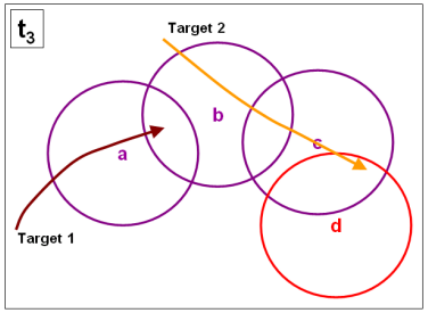

(c)

Fig. 4. Strategy 2: Illegal address and reapplying.

\subsection{Overview of DMTTP}

Before we explain the DMTTP protocol, we first make the following assumptions.

- Each sensor knows the neighbors whose sensing ranges overlap its own. It is not difficult to implement this assumption since, for most sensor nodes, communication range is much wider than sensing range.

- Targets are allowed to change their velocities and directions at any time. In addition, target sizes are assumed to be negligible to make sure that a target cannot be located in two positive cliques at the same time.

- The location of each sensor is known and stationary. This assumption can be reasonable since many prior works [14][15] have studied the location problem in sensor networks. Note that in our method the location information is only used in target trajectory estimation and is not necessary in target counting.

To attain the main purpose of maintaining every target address in time, the message containing three items, which are LastTrigSenSN, TarAddr, and SpSenStr, needs to be updated and exchanged between sensors. It is used for both target counting and trajectory estimation, and is stored in either Message Table or temp_Message Table (in sensor's memory). To increase tracking efficiency and decrease energy consumption, all processes in DMTTP are finished by only communicating with neighbors.

\subsection{Target Trajectory Estimation}

In this section, we present a method with focus on the ideal sensing model, based on target address for estimating target trajectory in a binary sensor network. In the ideal sensing model, if a target appears at a distance less than or equal to $r$ (the sensing range) from the sensor, it can be detected with 100\% probability. This model has a sharp 
sensing boundary; thus, it can simplify the problem that needs to consider sensing range. Nevertheless, the sensing range of a sensor is, in practice, not a perfect disk due to two factors, noise and power supply. To be more realistic, a probabilistic sensing model, where the detection probability decays with increased distance, is feasible. In this paper, we adopt an exponential model, which possesses the property that the detection probability of a target, which appears at a distance larger than $r$, degrades according to an exponential distribution, and achieves $100 \%$ probability when the target appears within a specific distance, $r$. In the following, the proposed target trajectory estimation algorithm is described. For a sensor with an ideal sensing range, it is obvious that a target must locate at a point within the sensing range of the sensor when the target triggers it. Since the neighbors of the triggered sensor do not change their states, the range of the target's location can be contracted into an arc $A$, which is given as:

$$
A=B_{x}-\bigcup R_{i}, \quad i \in x^{\prime} \text { s neighbors }
$$

where $B_{x}$ is the boundary of the sensing range of a triggered sensor $x$ and $R_{i}$ is the sensing range of a sensor $i$. The range of $A$, however, is still too rough to accurately discover a target trajectory. Hence, we present a technique to estimate the target location when a sensor is triggered.

Consider a target moving in a region, which contains the joint sensing ranges of two sensors, and crossing their boundaries in turn. Depending on DMTTP, the information regarding which sensor is triggered by which target is known.

We first compute the expected trajectory length between the arc $A$ of the last triggered sensor and that of the current one as:

$$
d_{e}=\int_{\theta_{1}}^{\theta_{2}} \int_{\phi_{1}}^{\phi_{2}} \frac{\sqrt{\left(\left(x_{1}+r \cos \theta\right)-\left(x_{2}+r \cos \phi\right)\right)^{2}+\left(\left(y_{1}+r \sin \theta\right)-\left(y_{2}+r \sin \phi\right)\right)^{2}}}{\left(\theta_{2}-\theta_{1}\right) \times\left(\phi_{2}-\phi_{1}\right)} d \phi d \theta .
$$

Note that since the probabilities of overestimating or underestimating the true trajectory length are the same, the greater the distance of movement of the target, the greater the accuracy of the estimation.

Subsequently, we compute the distance between a target and a sensor whose sensing range the target enters. Since the target can enter from any point at any angle on the $\operatorname{arc} A$ of a sensor, we arbitrarily select a point $p$ as the entrance, and the target will locate at any point of a $d_{e}$-unit-radius ring centered at $p$ inside the sensor's sensing range. Let $\omega$ be the incident angle bounded by $\omega_{\max }$, which is $\cos ^{-1}(d / 2 r)$. Then, given that $s_{e}$ denotes the expected distance between a sensor and a target inside its sensing range, we can attain:

$$
s_{e}=\sqrt{\frac{d_{e}}{4}\left(2 d_{e}-\frac{\sqrt{4 r^{2}-d_{e}^{2}}}{\omega_{\max }}\right)} .
$$

Finally, we choose a point on $A$ corresponding to the triggered sensor, which has a distance to another sensor closer to $s_{e}$. This point is recognized as a target position at the moment when the target triggers a sensor.

\section{Conclusion}

We study the performance limit of binary sensor networks in counting multiple targets in a two-dimensional sensing field using spatial information only. We propose a distributed multiple target tracking protocol (DMTTP) to solve two challenging problems, caused by the use of spatiotemporal information, for target counting and trajectory estimation, without resorting to solving minimum clique partitions that is NP-complete.

\section{References}

[1] X. Ji, H. Zha, J. Metzner, and G. Kesidis, "Dynamic cluster structure for object detection and tracking in wireless adhoc sensor networks," Proc. ICC, June 2004. 
[2] W.-P. Chen, J. C. Hou, and L. Sha, "Dynamic clustering for acoustic target tracking in wireless sensor networks," IEEE Transactions on Mobile Computing, 3(3):258-271, 2004.

https://doi.org/10.1109/TMC.2004.22

[3] J. Shin, L. Guibas, and F. Zhao, "A distributed algorithm for managing multi-target identities in wireless ad-hoc sensor networks," Proc. IPSN, April 2003.

https://doi.org/10.1007/3-540-36978-3_15

[4] S.Gandhi, R. Kumar, and S. Suri, "Target Counting under Minimal Sensing: Complexity and Approximations," Proc. ALGOSENSORS, 2008.

https://doi.org/10.1007/978-3-540-92862-1_4

[5] T. Roosta, M. Mishra, and A. Ghazizadeh, "Robust estimation and detection in ad hoc and sensor networks," Proc. IEEE MASS, October 2006.

https://doi.org/10.1109/MOBHOC.2006.278562

[6] A.O. Ercan, A.E. Gamal, and L. Guibas, "Object tracking in the presence of occlusions via a camera network," Proc. IPSN, April 2007.

https://doi.org/10.1145/1236360.1236425

[7] C. Meesookho, S. Narayanan, and C. S. Raghavendra, "Collaborative classification applications in sensor networks," Proc. Workshop on Sensor Array and Multichannel Signal Processing, August 2002.

https://doi.org/10.1109/SAM.2002.1191063

[8] I. F. Akyildiz, W. Su, Y. Sankarasubramaniam, and E. Cayirci, "A survey on sensor networks," IEEE Communications Magazine, 40:102-114, Aug. 2002.

https://doi.org/10.1109/MCOM.2002.1024422

[9] J. Aslam, Z. Butler, F. Constantin, V. Crespi, G. Cybenko, and D. Rus, "Tracking a moving object with a binary sensor network," Proc. ACM SenSys, November 2003.

https://doi.org/10.1145/958491.958509

[10] W. Kim, K. Mechitov, J.-Y. Choi, and S. Ham, "On target tracking with binary proximity sensors," Proc. IPSN, 2005.

[11] N. Shrivastava, R. Mudumbai, U. Madhow, and S. Suri, "Target tracking with binary proximity sensors: Fundamental limits, minimal descriptions, and algorithms," Proc. ACM SenSys, October 2006.

https://doi.org/10.1145/1182807.1182833

[12] M. M. Morelande, "Tracking multiple targets with a sensor network," Proc. the 9th International Conf. Information Fusion, July 2006.

https://doi.org/10.1109/ICIF.2006.301697

[13] J. Singh, U. Madhow, r. Kumar, S. Suri, and R. Cagley, "Tracking multiple targets using binary proximity sensors," Proc. IPSN, April 2007. https://doi.org/10.1145/1236360.1236427

[14] L. Doherty, K.S.J. Pister, and L.E. Ghaoui, "Convex position estimation in wireless sensor networks," Proc. IEEE INFOCOM, April 2001. https://doi.org/10.1109/INFCOM.2001.916662

[15] A. Savvides, C.-C. Han, and M.B, "Strivastava. Dynamic fine-grained localization in ad hoc networks of sensors," Proc. ACM MobiCom, July 2001.

[16] S.C. Chang, D.I. Cheng, and M. Marek-Sadowska, "Minimizing ROBDD size of incompletely specified multiple output functions," Proc. European Design and Test Conf. (EDTC), 1994. 
https://doi.org/10.1109/EDTC.1994.326921

[17] J. Gramm, J. Guo, F. Hüffner, and R. Niedermeier, "Data reduction, exact, and heuristic algorithms for Clique Cover," Proc. Workshop on Algorithm Engineering and Experiments (ALENEX), 2006.

https://doi.org/10.1137/1.9781611972863.9

[18] M. R. Morelande, "Tracking multiple targets with a sensor network," Proc. International Conference on Information Fusion, July 2006.

https://doi.org/10.1109/ICIF.2006.301697 